\title{
Challenging new targets for CNS-HIV infection
}

\author{
Graduate School of Nanotechnology, University of Trieste, Trieste, Italy \\ ${ }^{2}$ School of Medicine, University of Trieste, Trieste, Italy \\ ${ }^{3}$ School of Medicine, University of Cagliari, Cagliari, Italy
}

Mario Ganau ${ }^{1}$, Lara Prisco², Daniele Pescador ${ }^{2}$ and Laura Ganau ${ }^{3}$

Edited by:

Nick Andrews, Pfizer, UK

\section{Reviewed by:}

Muzamil Ahmad, Indian Institute of

Integrative Medicine, India

Jason B. Wu, Cedars-Sinai Medical

Center, USA

\section{*Correspondence:}

Mario Ganau, Graduate School of Nanotechnology, University of Trieste, Via San Francesco 12, 34133 Trieste, Italy.

e-mail: mario.ganau@phd.units.it
The central nervous system (CNS) represents an important target for HIV infection during multiple stages of the disease: early, after invasion of the host, acting as a viral reservoir; lately, subverting its function and causing peripheral neuropathies and neurocognitive disorders; and lastly, during the final stage of NeuroAIDS, triggering opportunistic infections, cancers, and dementia. Highly active antiretroviral therapy, a combination of drugs that inhibits enzymes essential for HIV replication, can reduce the viremia and the onset of opportunistic infections in most patients, and prolong the survival. Among the limits of the current treatments the most noticeable is the inability to eradicate HIV-infected cells, both, limiting the time frame in which antiretroviral therapies initiated after exposure to HIV can prevent infection, and allowing replication-competent virus that persists in infected cells to emerge rapidly after the cessation of treatments. Many strategies are currently under evaluation to improve HIV treatment, unfortunately more than $98 \%$ of drug candidates for CNS disorders never make it to the clinic; here in we report how nanoformulated strategies might be adapted and applied to the field of CNS-HIV infection.

Keywords: CNS-HIV infection, NeuroAIDS, nanoformulated HAART

\section{BACKGROUNG}

The central nervous system (CNS) represents an important target for HIV infection during multiple stages of the disease: early, after invasion of the host, since the virus rapidly enters the CNS, which then constantly acts as a viral reservoir; lately, subverting its function and causing peripheral neuropathies and neurocognitive disorders; and lastly, during the final stage of NeuroAIDS, triggering opportunistic CNS infections, cancers, and dementia (Tardieu and Boutet, 2002). Highly active antiretroviral therapy (HAART), a combination of drugs that inhibits enzymes essential for HIV replication, can reduce the viremia and the onset of opportunistic infections in most patients, and prolong the survival (see Table 1). Although HAART has reduced the incidence of clinical signs of neurological disease in HIV-infected individuals, autopsy studies suggest that there has been no corresponding decline in the incidence of inflammatory lesions in the CNS.

Among the limits of the current treatments the most noticeable is the inability to eradicate HIV-infected cells, both, limiting the time frame in which HAART initiated after exposure to HIV can prevent infection, and allowing replication-competent virus that persists in infected cells to emerge rapidly after the cessation of HAART. According to those considerations, there is evidence that HAART is less effective in lowering virus replication in the CNS than in the blood (Gisolf et al., 2000); and HAART resistant viruses are more often found in the cerebrospinal fluid (CSF) than in biological samples (Cinque et al., 2001). The principal challenges in drug development for CNS-HIV infections are: (1) understanding the pathways for viral entry, replication, reservoirs, and glial activation in order to identify new targets for treatment, (2) developing selective therapeutics agents for those targets, or shaping effective strategies to deliver them within the CNS, and finally but not to underestimate (3) collaborating with pharmaceutical companies to test promising drugs in clinical trials, and eventually exploit them in clinical practice.

\section{CURRENT UNDERSTANDING OF CNS-HIV INFECTION}

HIV is known to productively replicate in macrophages and microglia of the CNS, but it also infects, via a CD4-independent pathway, macroglial cells such as astrocytes and most-importantly brain microvascular endothelial cells, which represent the major component of the blood brain barrier (BBB; Bissel and Wiley, 2004). Although it is generally accepted that neurons do not become infected by HIV, recent reports have shown that neuronal progenitor brain cells can be infected by various viral strains (i.e., HIV-1), eventually playing a role as viral reservoirs (Lawrence et al., 2004). Moreover, one of the important features of CNS infection is that its viral populations can diverge from those of plasma: indeed, genetic compartmentalization of CSF HIV-1 infection has been nicely demonstrated in a series of studies using the heteroduplex tracking essay to compare blood and CSF viral populations crosssectionally and longitudinally (Ritola et al., 2005); therefore even if during acute infection the two population are identical, during chronic infection and especially in cases of HIV encephalitis they genetically and functionally diverge, and may justify the failure of antiretroviral therapy (Strain et al., 2005).

Identification of viral and cellular mechanisms in the CNS that control virus replication after acute infection, maintain latency during the asymptomatic stage, and mediate resurgence of virus replication and development of HIV-associated encephalitis during late infection, is critical. The dynamic signaling pathways that are activated by virus replication and active/infiltrating macrophages and lymphocytes and the cytokines, chemokines, and neurotoxic products they elaborate may interfere with the homeostatic signaling pathways that maintain normal quiescent 
Table 1 | HAART currently in use in the clinical practice.

\begin{tabular}{|c|c|}
\hline Classes of drugs & Phase of the retroviral life-cycle targeted \\
\hline Entry inhibitors (Els) & They interfere with binding, fusion, and entry of HIV-1 to the host cell \\
\hline CCR5 receptor antagonists (CCR5RAs) & $\begin{array}{l}\text { They bind to the CCR5 receptor on the surface of the T-Cell and block viral attachment to the } \\
\text { cell. If HIV cannot attach to the cell, it cannot gain entry to replicate }\end{array}$ \\
\hline $\begin{array}{l}\text { Nucleoside reverse transcriptase inhibitors (NsRTIs) and } \\
\text { nucleotide reverse transcriptase inhibitors (NtRTIs) }\end{array}$ & $\begin{array}{l}\text { They inhibit reverse transcription by being incorporated into the newly synthesized viral DNA } \\
\text { strand as faulty nucleotides; they both act as competitive substrate inhibitors }\end{array}$ \\
\hline Non-nucleoside reverse transcriptase inhibitors (NNRTIs) & $\begin{array}{l}\text { They inhibit reverse transcriptase by binding to an allosteric site of the enzyme; NNRTIs act as } \\
\text { non-competitive inhibitors of reverse transcriptase }\end{array}$ \\
\hline Protease inhibitors (PIs) & $\begin{array}{l}\text { They target viral assembly by inhibiting the activity of protease, an enzyme used by HIV to } \\
\text { cleave nascent proteins for the final assembly of new virions }\end{array}$ \\
\hline Integrase inhibitors (IIs) & $\begin{array}{l}\text { They inhibit the enzyme integrase, which is responsible for integration of viral DNA into the } \\
\text { DNA of the infected cell }\end{array}$ \\
\hline Maturation inhibitors (Mls) & $\begin{array}{l}\text { They inhibit the last step in gag processing in which the viral capsid polyprotein is cleaved, } \\
\text { thereby blocking the conversion into mature capsid protein }\end{array}$ \\
\hline
\end{tabular}

brain function. To understand the correlates in terms of neuroprotection, neuroinflammation, and neurodegeneration processes many preclinical models have been developed, such as: HIV transgenic rat (i.e., tg rat from Reid et al., 2001), or mice (i.e., gp 120 tg mice from Toggas et al., 1994), but also those obtained by injection of HIV proteins (i.e., gp 120 from Sundar et al., 1991; or Tat and Bansal et al., 2000), or HIV-infected macrophages into the brain of mice (Anderson et al., 2003). The most studied signaling pathways include, among others, the GSK3 $\beta$, CDK5, ERK, Notch, p38, and JNK cascades. For instance, it is noteworthy that many mitogen-activated protein kinases are pivotal during acute, asymptomatic, and terminal infection: some of them seems to promote cell growth and differentiation in response to initial infection (i.e., ERK), whereas others are associated to growth arrest, apoptosis, and oncogenic transformation during AIDS. Those data might explain some peculiar characteristics of the HIV-CNS infection: in contrast to HIV-infected CD4+ T cells, infection in brain macrophages and microglial cell lines leads to an extended life span and elevated survival against apoptotic stresses (Chugh et al., 2007), and only during terminal infection, when there is resurgent virus replication, the balance favors neuronal death (i.e., via activation of JNK and p38) reflecting a failure to maintain homeostasis in the CNS (Barber et al., 2004) with the consequent motor and cognitive impairments.

\section{DEVELOPING STRATEGIES}

Many strategies are currently under evaluation to improve HIV treatment, but a relatively small number are primarily dedicated to the alleviation of neurological complaints or the control of NeuroAIDS, and unfortunately more than $98 \%$ of drug candidates for CNS disorders never make it to the clinic (Pardridge, 2003). Herein are a couple of examples picturing how researches, initially conducted for neoplastic, neurodegenerative, or epileptic disorders, on specific pathways and delivery systems, might be adapted and applied to the field of CNS-HIV infection (see Figure 1).

\section{INNOVATIVELY TARGETED PATHWAYS}

Discovered in 1898, mitoguazone (MGBM) had been investigated but abandoned as an anti-cancer drug, recently it was found to cause selective loss of CD16+ HIV-infected cells, specifically by blocking osteopontin needed to transform a circulating macrophage into a CNS/resident one. MGBG although ineffective in reducing HIV viremia, does appear to shut down viral evolution by selectively killing recent migrants macrophages rather than resident cells, and to be safe over the long-term treatment in AIDS patients (Jeymohan et al., 2009).

The manipulation of IFN $\alpha$ and IFN $\gamma$ APOBEC pathways could prevent HIV invasion of CNS because of the potent intrinsic immunity exerted by the expression of APOBEC3G gene in microvascular endothelial cells (Argyris et al., 2007). This gene code for proteins possessing cytidine deaminase activity and allow the BBB to actively neutralize the effects of viral infectivity factors (Vif), which are essentials for viral entry and spread into the CNS (Jeong et al., 2008).

NK-1, also known as Substance P, was the first neuropeptide discovered in 1931, it is widely distributed in the central and peripheral nervous system where it functions as a potent mediator of immunoregulation. Agonists to NK-1 receptor potently inhibit both in vitro and in vivo HIV replication in human macrophages, apparently down regulating CCR5 (Jeymohan et al., 2009).

Initially recognized, and extensively studied as a promising target for anti-cancer therapies because of its role during tumorigenesis, the PI3K/Akt cell survival pathway became a target for anti-HIV treatment after the observation that PI3K inhibitors (such as wortmannin and LY294002) or Akt inhibitors (such as miltefosine), both able to cross the BBB, contrast the cytoprotective effect exerted by Tat proteins over macrophage/microglial cells, and make them again susceptible to cell death following an apoptotic stimulus (Chugh et al., 2007, 2008). Most interestingly $\mathrm{PI} 3 \mathrm{~K} /$ Akt inhibitors proved to be effective without harming uninfected cells and therefore experimental data as far available support their possible use for HIV-therapy and targeting of long-lived viral reservoirs.

\section{NANOFORMULATED HAART}

Nanoformulated HAART essentially manipulates the immune system for therapeutic purposes by using synthetic or biological nanoengineered drug carriers. Those nanoformulated drugs have been proven to be efficient delivery vehicles for a wide range of therapeutic agents, and can be easily injected in the blood 


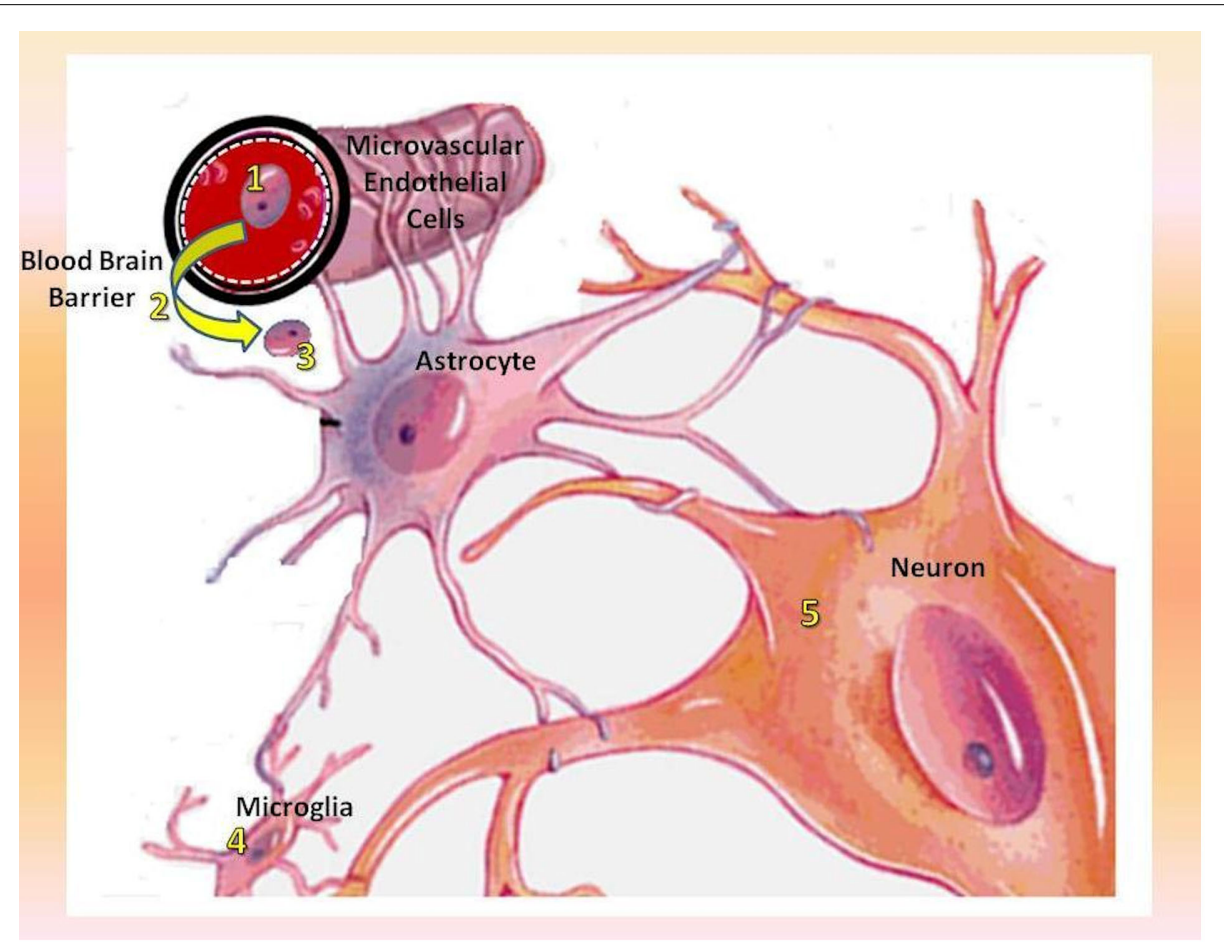

FIGURE 1 | Mechanism of CNS-HIV infection along with the relative therapeutic targets. 1. Selective killing of migrant macrophages (MGBM); 2. neutralization of Vif on microvascular endothelial cells (IFN $\alpha$ and IFN $\gamma$ ) and subsequent inhibition of viral entry into the CNS; 3. inhibition of HIV replication in macrophages and viral spread into the CNS (NK-1-agonists);
4. inhibition of cytoprotective effect exerted by Tat proteins over microglial cells which act as CNS viral reservoirs (PI3K and Akt inhibitors); 5. protection of neural integrity and plasticity along with prevention of neurotoxicity and neuronal death (glutamate receptors-, GSK3 $\beta-$, JNK-, and p38-inhibitors). circulation or virtually into any site of the brain with minimal invasiveness. Major concerns to this therapeutic approach are the choice of drug administration (systemic or local) and consequently the residence in the systemic blood circulation, or in the brain tissue adjacent to topic injection, the drug loading efficiency, and the burst release effect. Furthermore, many other question marks are still to be answered, or even formulated.

Concerning synthetic carriers, hyaluronic acid, a linear polysaccharide composed of alternating D-glucuronic acid and $N$ acetyl-D-glucosamine units, because of its immunoneutrality, has been proposed as a biocompatible and biodegradable biomaterial for tissue engineering, and in drug delivery systems into the CNS: particularly appealing would seem the opportunity to produce drug-incorporated hyaluronic acid nanoparticles (Jeong et al., 2008). As well monocytes, because of their abilities to move drugs inside the cells have advantages as a depot for HAART, but also they might improve compliance as drugs can be released from cells for periods up to weeks or longer. Nevertheless, further application of biological nanoengineered drug carriers will require optimization of nanoparticles uptake, deeper knowledge of their kinetics across the BBBs, and more sophisticated imaging systems to track migration of monocyte-macrophages to the brain and target the delivery of specific therapeutics (Jeymohan et al., 2009).

\section{NEUROPROTECTIVE STRATEGY}

Beside compounds aimed at control the virus, others try to treat the effects of the underlying neuroinflammation and neurodegeneration mechanisms, and might provide a beneficial supplement to HAART.

Neurotoxicity is mainly mediated through glutamate production, which is triggered by NMDA subtypes of glutamate receptors, and correlated to caspase activation and subsequent neuronal apoptosis (Erdmann et al., 2006); accordingly, pharmacologic inhibition of glutaminase directly correlate to neuronal survival (Tian et al., 2008). Those insights support the hypothesis that inhibition of glutamate production by blocking mitochondrial glutaminase activity may clinically prevent neurotoxicity during HIV-1 infection.

Taken together the GSK3 $\beta$, CDK5, JNK, and p38 signaling pathways are important regulators of neurotoxicity, being responsible for synaptic and dendritic damage to pyramidal neurons, loss of immunoreactive neurons, and demyelinization. GSK3 $\beta$ inhibitors (i.e., lithium, valproic acid) as well as CDK5 inhibitors (i.e., roscovitine) seems to protect neuronal integrity and plasticity (Crews et al., 2009), while JNK- and p38-inhibitors (i.e., minocycline) may prevent neuronal death (Lin et al., 2001; Bozyczko-Coyne et al., 2002) each of those target holds promise for the development of treatment strategies to ameliorated the neuropathological effects exerted by HIV proteins (Tat, Vif, etc.).

\section{CONCLUSION}

Combinations of drugs work better and longer than seen previously, patients benefit demonstrably from the magnitude and duration of viral suppression, and require more and more symptomatic treatment of NeuroAIDS cognitive impairment. 
However HAART is very demanding for patients, non-adherence can lead to therapeutic failure, and in addition drug resistance is a continuing problem; therefore, the need for improved drug and delivery systems, but also an interest for adaptive treatment strategies and mathematical modeling to manage structured treatment interruptions (Rosenberg et al., 2007), have been outlined by many researchers. To this regard a thigh collaboration with industry is strictly required and introduces a new set of challenges such as: (1) circulation of promising ideas, often just developed with university grants, (2) protection of intellectual property and patent rights, (3) fund-raising opportunities for start-ups during preclinical researches on novel compounds, until (4) identification of potential blockbusters appealing to

\section{REFERENCES}

Anderson, E. R., Boyle, J., Zink, W. E., Persidsky, Y., Gendelman, H. E., and Xiong, H. (2003). Hippocampal synaptic dysfunction in a murine model of human immunodeficiency virus type 1 encephalitis. Neuroscience 118, 359-369.

Argyris, E., Acheampong, E., Wang, F., Huang, J., Chen, K., Mukhtar, M., and Zhang, H. (2007). The IFNinduced expression of APOBEC3G in human blood brain barrier exerts a potent intrinsic immunity to block HIV-1 entry to central nervous system. Virology 25, 440-451.

Bansal, A. K., Mactutus, C. F., Nath, A., Maragos, W., Hauser, K. F., and Booze, R. M. (2000). Neurotoxicity of HIV-1 proteins gp120 and Tat in the rat striatum. Brain Res. 879 , 42-49.

Barber, S. A., Uhrlaub, J. L., DeWitt, J. B., Tarwater, P. M., and Zink, M. C. (2004). Dysregulation of mitogenactivated protein kinase signaling pathways in simian immunodeficiency virus encephalitis. Am. J. Pathol. 164, 355-362.

Bissel, S. J., and Wiley, C. A. (2004). Human immunodeficiency virus infection of the brain: pitfalls in evaluating infected/affected cell populations. Brain Pathol. 14, 97-108.

Bozyczko-Coyne, D., Saporito, M. S., and Hudkins, R. L. (2002). Targeting the JNK pathway for therapeutic benefit in CNS disease. Curr. Drug Targets CNS Neurol. Disord. 1, 31-49.

Chugh, P., Bradel-Tretheway, B., Monteiro-Filho, C., Planelles, V., Maggirwar, S. B., Dewhurst, S., and Kim, B. (2008). Akt inhibitors as an HIV-1 infected macrophage-specific anti-viral therapy. Retrovirology 5, 11.

Chugh, P., Fan, S., Planelles, V., Maggirwar, S. B., Dewhurst, S., and Kim, B. (2007). Infection of human immunodeficiency virus and intracellular viral Tat protein exert a pro-survival effect in human microglial cell line. J. Mol. Biol. 366, 67-81.

Cinque, P., Presi, S., Bestetti, A., Pierotti, C., Racca, S., Boeri, E., Morelli, P., Carrera, P., Ferrari, M., and Lazzarin, A. (2001). Effect of genotypic resistence on the virological response to highly active antiretroviral therapy in cerebrospinal fluid. AIDS Res. Hum. Retroviruses 17, 377-383.

Crews, L., Patrick, C., Achim, C. L., Everall, I. P., and Masliah, E. (2009). Molecular pathology of NeuroAIDS. Int. J. Mol. Sci. 10, 1045-1063.

Erdmann, N., Whitney, N., and Zheng, J. (2006). Potentiation of excitotoxicity in HIV-1 associated dementia and the significance of glutaminase. Clin. Neurosci. Res. 6, 315-328.

Gisolf, E. H., Enting, R. H., Jurriaans, S., de Wolf, F., van der Ende, M. E., Hoetelmans, R. M., Portegies, P., and Danner, S. A. (2000). Cerebrospinal fluid HIV-1 RNA during treatment with ritonavir/saquinavir or ritonavir/saquinavir/stavudine. AIDS 14, 1583-1589.

Jeong, Y. I., Kim, S. T., Jin, S. G., Ryu, H. H., Jin, Y. H., Jung, T. Y., Kim, I. Y., and Jung, S. (2008). Cisplatin-incorporated hyaluronic acid nanoparticles based on ioncomplex formation. J. Pharm. Sci. 97, 1268-1276.

Jeymohan, J., Clifford, D., Douglas, S. D., Fox, H., Gendelman, H. E., Gonzalez-Scarano, F., Grant, I., Major, E., McArthur, J., and NeuroAIDS Research Participants. (2009). Planning future strategies for domestic and international NeuroAIDS Research. J. Neuroimmune Pharmacol. 4, 283-297.

Lawrence, D. M., Durham, L. C., Schwartz, L., Seth, P., Maric, D., and Major, E. O. (2004). Human immunodeficiency virus type 1 infection of human brain-derived progenitor cells. J. Virol. 78, 7319-7328.

Big Pharma companies for introduction in the market and their clinical exploitation.

Finally, current criteria for initiation of HAART do not consider CNS outcomes as a benchmark of efficacy, thus establishing CNSbased criteria for initiation of protocols should be the ultimate goal not only for therapeutic but also for neuroprotection strategies; as recently stated by Jeymohan et al. (2009) on behalf of the NeuroAIDS research participants these priorities should be seen as a template for future growth of the field and its long-term impact.

\section{ACKNOWLEDGMENTS}

We are grateful to Mrs. Gloria Mognon for revision of the language and editing of the manuscript in its final version.

Lin, S., Zhang, Y., Dodel, R., Farlow, M. R., Paul, S. M., and Du, Y. (2001). Minocycline blocks nitric oxideinduced neurotoxicity by inhibition p38 MAP kinase in rat cerebellar granule neurons. Neurosci. Lett. 315, 61-64.

Pardridge, W. M. (2003). Blood-brainbarrier drug delivery: the future of brain drug development. Mol. Interv. 3, 90-105.

Reid, W., Sadowska, M., Denaro, F., Rao, S., Foulke, J. Jr., Hayes, N., Jones, O., Doodnauth, D., Davis, H., Sill, A., O’Driscoll, P., Huso, D., Fouts, T., Lewis, G., Hill, M., Kamin-Lewis, R. Wei, C., Ray, P., Gallo, R. C., Reitz, M., and Bryant, J. (2001). An HIV1 transgenic rat that develops HIVrelated pathology and immunologic dysfunction. Proc. Natl. Acad. Sci. U.S.A. 98, 9271-9276.

Ritola, K., Robertson, K., Fiscus, S. A., Hall, C., and Swanstrom, R. (2005). Increased human immunodeficiency virus type 1 (HIV-1) env compartmentalization in the presence of HIV-1 associated dementia. J. Virol. 79, 10830-10834.

Rosenberg, E. S., Davidian, M., and Banks, H. T. (2007). Using mathematical modeling and control to develop structured treatment interruption strategies for HIV infection. Drug Alcohol Depend. 88(Suppl. 2), S41-S51.

Strain, M. C., Letendre, S., Pillai, S. K., Russell, T., Ignacio, C. C., Günthard, H. F., Good, B., Smith, D. M., Wolinsky, S. M., Furtado, M. Marquie-Beck, J., Durelle, J., Grant, I., Richman, D. D., Marcotte, T., McCutchan, J. A., Ellis, R. J., and Wong, J. K. (2005). Genetic Composition of human immunodeficiency virus type-1 in cerebrospinal fluid and blood without treatment and during failing antiretroviral therapy. J. Virol. 79, 1772-1788.

Sundar, S., Cierpial, M., Kamaraju, L. Long, S., Hsieh, S., Lorenz, C., Aaron, M., Ritchie, J. C., and Weiss, J. M.
(1991). Human immunodeficiency virus glycoprotein (gp120) infused into rat brain induces interleukin 1 to elevate pituitary-adrenal activity and decreased peripheral cellular immune responses. Proc. Natl. Acad. Sci. U.S.A. 88, 11246-11250.

Tardieu, M., and Boutet, A. (2002). HIV1 and the central nervous system. Curr. Top. Microbiol. Immunol. 265, 183-195.

Tian, C., Erdmann, N., Zhao, J., Cao, Z. Peng, H., and Zheng, J. (2008). HIVinfected macrophages mediate neuronal apoptosis through mitochondrial glutaminase. J. Neurochem. 105, 994-1005.

Toggas, S., Masliah, E., Rockenstein, E., Rall, G. F., Abraham, C. R., and Mucke, L. (1994). Central nervous system damage produced by expression of the HIV-1 coat protein gp120 in transgenic mice. Nature 367, 188-193.

Conflict of Interest Statement: The authors declare that the research was conducted in the absence of any commercial or financial relationships that could be construed as a potential conflict of interest.

Received: 08 January 2012; accepted: 06 March 2012; published online: 23 March 2012.

Citation: Ganau M, Prisco L, Pescador $D$ and Ganau L (2012) Challenging new targets for CNS-HIV infection. Front. Neur. 3:43. doi: 10.3389/fneur.2012.00043

This article was submitted to Frontiers in Neuropharmacology, a specialty of Frontiers in Neurology.

Copyright (C) 2012 Ganau, Prisco, Pescador and Ganau. This is an openaccess article distributed under the terms of the Creative Commons Attribution Non Commercial License, which permits non-commercial use, distribution, and reproduction in other forums, provided the original authors and source are credited. 